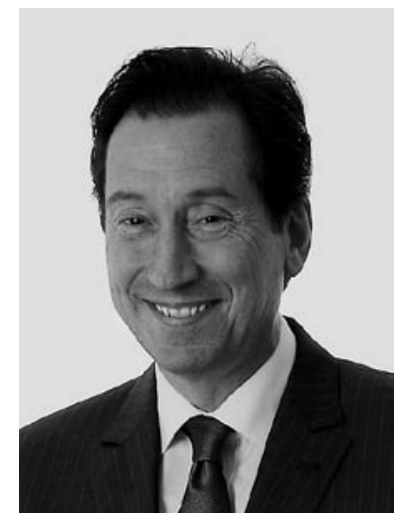

Jürgen Weber

\section{Nachhaltigkeit als Innovation}

Die Wirtschaftskrise hat die Controller derzeit voll im Griff. 20 - 30 \% Mehrbelastung sind für sie keine Ausnahme. Auf allen Feldern des Controllings arbeiten Controller seit Monaten intensiver und länger. Es zeigt sich aber auch, dass Controller in der Krise nicht den Mut haben, Grundsätzliches zu verändern - auch wenn sie die Notwendigkeit hierzu sehen. Noch fehlt der Schritt, die gewonnenen Erkenntnisse in konkretes Handeln umzusetzen.

Wenn schon nicht die Krise als Anstoß reicht: Wie halten es Controller generell mit Veränderung und Innovation? Ohne der Zunft zu nahe zu treten, lässt sich sicher feststellen, dass in der Vergangenheit beide Themen im "mind set" der Controller keine Priorität besaßen. Pläne sind ihr Einmaleins. Viele sehen ihre primäre Aufgabe darin, einen gegebenen Rahmen zu optimieren. Innovationen, die ganz neue Optionen versprechen und/oder Spielregeln im Markt verändern wollen, bereiten ihnen Probleme. Der Grund hierfür ist schnell gefunden. Solche Neuerungen sind nicht wirklich planbar. Bei aller anfänglichen Euphorie bleibt ein großer Teil von ihnen lediglich eine spannende Idee. Controller sind eher Bewahrer denn Innovatoren.

Bewährte Aufgaben beizubehalten, muss nicht falsch sein. Dennoch stellt sich die Frage: Was war die letzte Innovation der Controller? Die Balanced Scorecard liegt lange zurück, das Thema Wertorientierung ebenfalls. Beide Konzepte haben sich zudem als wenig nachhaltig erwiesen - und da sind wir bei einem guten Stichwort angelangt. Nicht nur die Krise wird dazu führen, dass sich die Unternehmenssteuerung nachhaltig verändern wird; Nachhaltigkeit selbst ist der Grund für einen solchen Veränderungsbedarf. Ausgelöst durch die weltweite Klimadebatte hat das Thema längst das Stadium des Idealistischen sowie Esoterischen überwunden und ist mit konkreten wirtschaftlichen Implikationen verbunden.

Nachhaltigkeit fehlt aber bisher auf der Agenda vieler Controller. Das sollte sich möglichst schnell ändern. Wie eben angedeutet, werden die wirtschaftlichen Konsequenzen immer deutlicher sichtbar. Das Thema muss bereits in der Produktgestaltung Berücksichtigung finden ( $\mathrm{CO}_{2}$-arme Konstruktion), führt zu neuen Produkten (wie etwa die go green-Produkte der DHL), darf nicht bei der Gestaltung der Lieferantenstruktur fehlen und reicht bis in spezielle Marketing-Strategien. Damit Nachhaltigkeit wirklich nachhaltig im Unternehmen verankert wird, muss es in die Regelprozesse der Steuerung integriert werden. Ähnliches gilt für die Informationssysteme (",Carbon Accounting"). Damit ist Nachhaltigkeit ein Feld, das auch und gerade die Controller betrifft. Sie sollten nicht warten, bis sie zum Handeln aufgefordert werden. Sie können vielmehr beweisen, dass sie innovativ sind. Die Chance, ihre Manager durch proaktives Handeln für sich zu gewinnen, sollten sie nicht verstreichen lassen. Ergreifen sie diese nicht, werden sich andere Stellen im Unternehmen des Themas annehmen - diese potenzielle Konkurrenz kann Controllern nicht recht sein!

Ziel dieses Heftes ist es, Ihnen einen fundierten Überblick über das Thema Nachhaltigkeit zu verschaffen. Durch das Studium der inhaltlich breit gefächerten Beiträge werden Sie zumindest in die Lage versetzt kompetent mitzudiskutieren - und das ist vielleicht der Beginn einer nachhaltigen Beschäftigung mit Nachhaltigkeit!

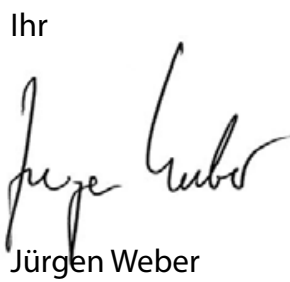

